\title{
Bayesian component separation: The Planck experience
}

\section{Ingunn Kathrine Wehus and Hans Kristian Eriksen}

Institute of Theoretical Astrophysics, University of Oslo, Postboks 1029 Blindern, 0315 Oslo, Norway

email: i.k.wehus@astro.uio.no

\begin{abstract}
Bayesian component separation techniques have played a central role in the data reduction process of Planck. The most important strength of this approach is its global nature, in which a parametric and physical model is fitted to the data. Such physical modeling allows the user to constrain very general data models, and jointly probe cosmological, astrophysical and instrumental parameters. This approach also supports statistically robust goodness-of-fit tests in terms of data-minus-model residual maps, which are essential for identifying residual systematic effects in the data. The main challenges are high code complexity and computational cost. Whether or not these costs are justified for a given experiment depends on its final uncertainty budget. We therefore predict that the importance of Bayesian component separation techniques is likely to increase with time for intensity mapping experiments, similar to what has happened in the CMB field, as observational techniques mature, and their overall sensitivity improves.
\end{abstract}

Keywords. Cosmology: cosmic microwave background, Galaxy: general, Methods: data analysis

\section{Introduction}

On May 14th 2009, ESA's cosmology cornerstone satellite mission Planck (Planck Collaboration I 2016) launched from French Guyana. For the next four years, the satellite made the deepest full-sky measurements of the microwave sky to date, observing in nine frequencies between 30 and $857 \mathrm{GHz}$. Based on these measurements, the Planck collaboration was able to constrain the parameters of our currently best $\Lambda$ CDM cosmological model to unprecedented accuracy (Planck Collaboration XIII 2016); measure the angular power spectrum of weak gravitational lensing with $40 \sigma$ significance (Planck Collaboration XV 2016); rule out all inflationary models predicting a high degree of non-Gaussianity (Planck Collaboration XVII 2016); and open up a whole new view of the various emission mechanisms in the Milky Way (Planck Collaboration X 2016), just to mention a few highlights. Today, Planck represents a standard reference in the field of cosmology, and is likely to remain so in the foreseeable future.

These achievements are the direct results of an intimate collaboration between hundreds of engineers and scientists spanning more than 25 years, resulting in a number of breakthroughs in both instrumentation and data analysis techniques. In this short note, we will discuss one specific example of such breakthroughs, namely that of Bayesian component separation (Eriksen et al. 2008; Planck Collaboration X 2016), on which we have been working for the last five years. The primary goal of this line of work is to establish the cleanest possible representation of the CMB sky signal based on some set of "dirty" frequency maps, which includes both cosmological signal, astrophysical foregrounds originating both from our own Milky Way and beyond, and instrumental systematics and noise. Of course, this challenge is the topic of many competing algorithms, and there is no time or space to review all of these methods here (see, e.g., Leach et al. 2008). 

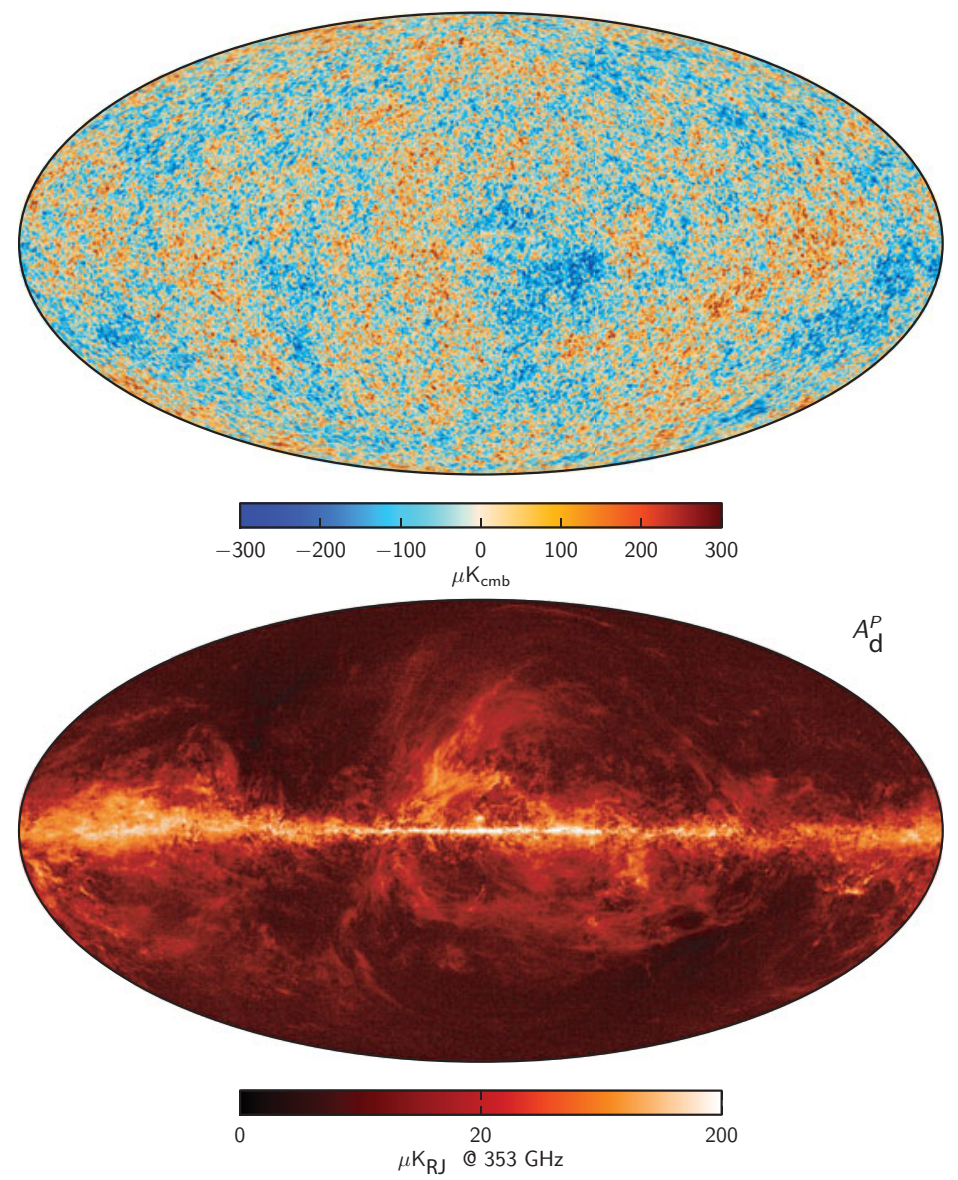

Figure 1. (Top:) The Planck 2015 Commander CMB temperature fluctuation map at $5^{\prime}$ FWHM angular resolution. (Bottom:) The Planck 2015 Commander thermal dust polarization amplitude map at $10^{\prime}$ FWHM resolution, evaluated at $353 \mathrm{GHz}$. Both plots are reproduced from Planck Collaboration I (2016).

What sets the Bayesian approach apart from most other techniques, though, is its insistence on defining a physically motivated data model, including both the signal of primary interest (for us, the CMB) and any contaminating contributions, whether it is from synchrotron or thermal dust emission, or from the instrument itself. This explicit parametric model is then fitted directly to the raw data in the posterior distribution sense using standard statistical Monte Carlo methods, such as Metropolis-Hastings or Gibbs sampling, or simply non-linear optimization. Thus, from a statistical point of view this framework represents a very direct and simple approach to component separation, as it almost exclusively uses methods that are routinely taught in introductory statistics courses. At the same time, the approach is also extremely bold, in that it aims to define a statistical description of the entire data set, and not only the primary signal of interest.

To make this point more explicit, let us consider the data model adopted for the latest Planck 2015 temperature analysis, as presented in Planck Collaboration X (2016),

$$
d_{\nu}(\theta)=g_{\nu} \sum_{i=1}^{N_{\text {com p }}} \mathcal{F}_{\nu}^{i}\left(\beta_{i}, \Delta_{\nu}\right) a_{i}+\mathcal{T}_{\nu} m_{\nu}+n_{\nu}
$$


Here $d_{\nu}$ denotes the observed data at frequency $\nu$; the sum runs over all relevant astrophysical components (e.g., CMB, synchrotron, free-free, spinning and thermal dust, and $\mathrm{CO}$ and other line emission mechanisms for the analysis in question), each of which is parameterized by some mixing matrix $\mathcal{F}_{\nu}^{i}$ with spectral parameters $\beta_{i}$, and an amplitude $a_{i}$ per pixel; $g_{\nu}$ and $\Delta_{\nu}$ represent instrumental parameters describing overall calibration and bandpass uncertainties, respectively; $\mathcal{T}_{\nu}$ indicates fixed templates with free amplitudes only, and accounts for zero-level and dipole uncertainties; and $\vec{n}_{\nu}$ denotes instrumental noise. In total, this model includes more than 300 million free and strongly correlated parameters, and jointly constraining these represents a major computational challenge. To this aim, we have over the last decade developed a computer code called Commander (Eriksen et al. 2004, 2008), which implements a particular MCMC sampling algorithm called Gibbs sampling that maps out the exact, full and complicated posterior distribution through iterative sampling over much simpler conditional distributions. This code has become a standard reference in the field, and many of the official Planck products have been derived with this code. Two particularly well-known examples are shown in Figure 1, namely the CMB temperature fluctuation map (top panel) and the thermal dust polarization amplitude map at $353 \mathrm{GHz}$ (bottom panel).

Based on the Planck experience, there is no question that the Bayesian approach is both powerful, flexible and highly productive. However, it is clearly also associated with a significant cost in terms of both manpower and computing resources, and future experimentalists may therefore ask themselves whether it makes sense to adopt an equally ambitious approach for their experiment. Giving some inputs to this question is the main goal of the current note.

\section{What has Bayes ever done for us?}

When the Bayesian approach to CMB component separation was first introduced in 2004 by Jewell et al. (2004), Wandelt et al. (2004) and Eriksen et al. (2004), the main motivation was to establish a coherent analysis framework that allowed for joint analysis of both CMB and astrophysical foreground signals, and, critically, to propagate their joint uncertainties from raw data products to final cosmological parameters. After going through the Planck experience, these ideas do remain important, and play important roles in the final results. However, even more important than these aspects for the overall final outcome of Planck is the insistence of physical modeling for all signal components.

For Planck, this point has had two important consequences. On the one hand, it implies that parameters that are normally considered "nuisance" parameters for cosmologists, such as thermal dust or CO emission maps, actually become valuable scientific products in their own rights. Furthermore, by virtue of being derived in precisely the same manner as the CMB signal, they are ensured the same high statistical confidence as the primary scientific target. As a result, the Commander approach delivered not only a CMB fluctuation map, but also individual estimates of synchrotron, free-free, spinning and thermal dust, and CO line emission (Planck Collaboration X 2016), all of which are used extensively by other groups.

On the other hand, there is in fact another aspect of the physical modeling approach that is even more important than the derivation of scientific ancillary products, and that is the method's unique ability to uncover instrumental systematic effects. To make this point explicit, we show in the left panel the publicly released Planck $2015353 \mathrm{GHz}$ map derived from the 353-2 bolometer alone (Planck Collaboration VIII 2016). By visual inspection, this map appears to be a very high signal-to-noise measurement of the true sky at $353 \mathrm{GHz}$, and no obvious artifacts may be seen. However, in the right column 

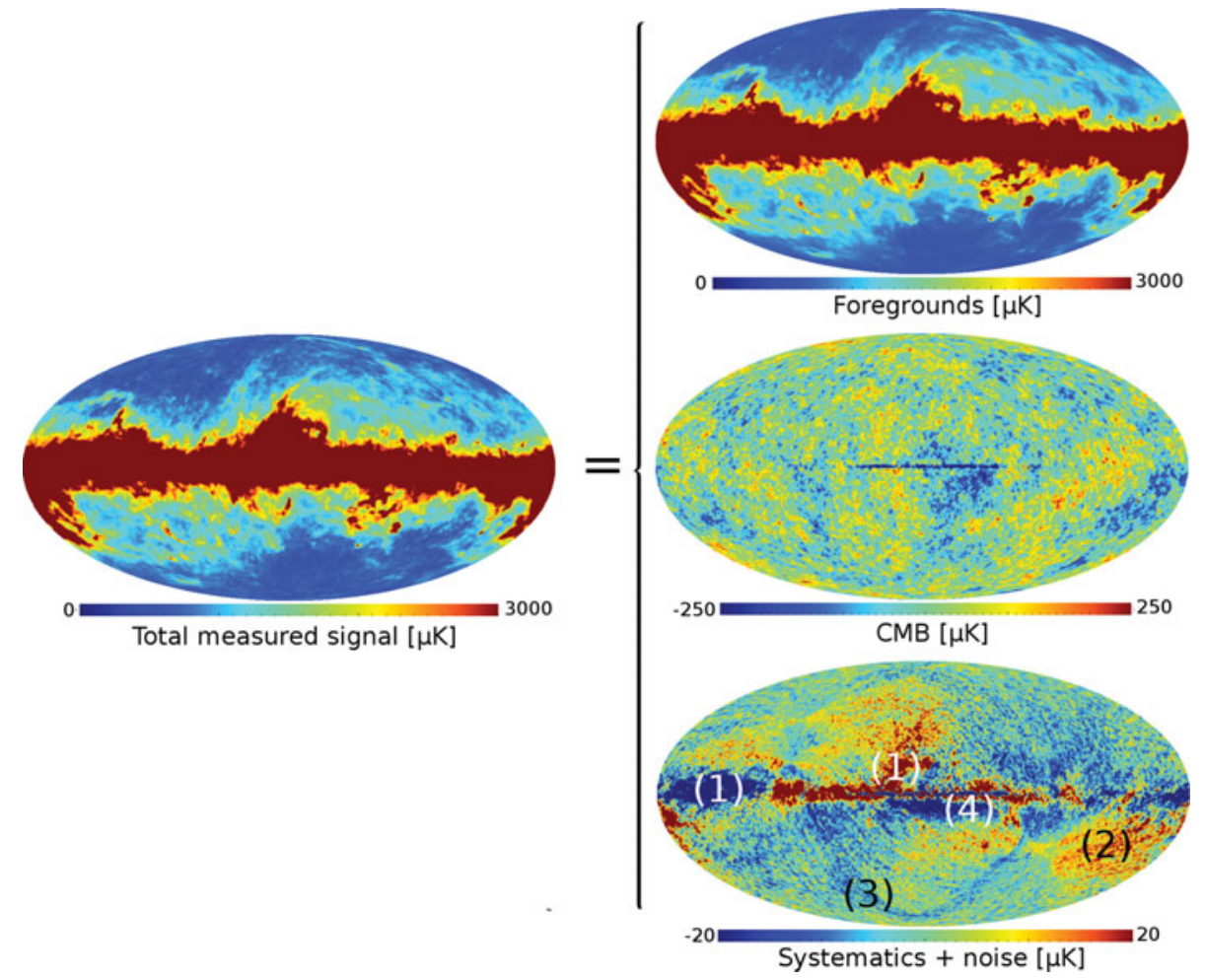

Figure 2. Decomposition of the Planck 2015 353-2 detector map (left panel) into Galactic foregrounds (right top panel), CMB fluctuations (right middle panel), and residual systematics and noise (right bottom panel). The latter three maps are all derived with Bayesian component separation, as implemented in Commander. The dominant instrumental systematic features seen in the bottom right panel correspond to (1) polarization-to-temperature leakage; (2) bandpass mismatch; (3) sidelobe pickup; and (4) transfer function mis-estimation. Note that none of these effects can be identified in the raw map, but they become visible only after component separation, when subtracting off a best-fit physical model.

we also show the (sum of the) astrophysical foregrounds at this channel (top panel), as estimated by Commander, and the corresponding CMB fluctuation map (middle panel). The bottom panel shows what we call the residual map, which is nothing but the difference between the data and the signal model, and encaptures unmodeled instrumental systematic effects and noise. Ideally, this map should look like uncorrelated noise with a local variance given by the observation time of the instrument in a given pixel, but clearly it does not. Instead, a wide range of residual instrumental systematic effects may be seen, and understanding and mitigating the features in this map is really where the vast majority of the analysis time for a given experiment is spent.

When looking at a residual map like this, the important goal is to understand each coherent feature physically; what causes it, and how can it be fixed? For instance, in our case the Galactic plane structures marked by (1) were shown to be correlated very strongly with the thermal dust polarization signal shown in the bottom panel of Figure 1, with a sign given by the detector orientation of the Planck detector. This effect could thus be traced down to polarization-to-temperature leakage through sub-optimal polarization reconstruction. In collaboration with the map making team, the issue could then be mitigated during low-level processing. Likewise, the diffuse structures marked by (2) were traced to bandpass differences between individual bolometers, leaking foreground 

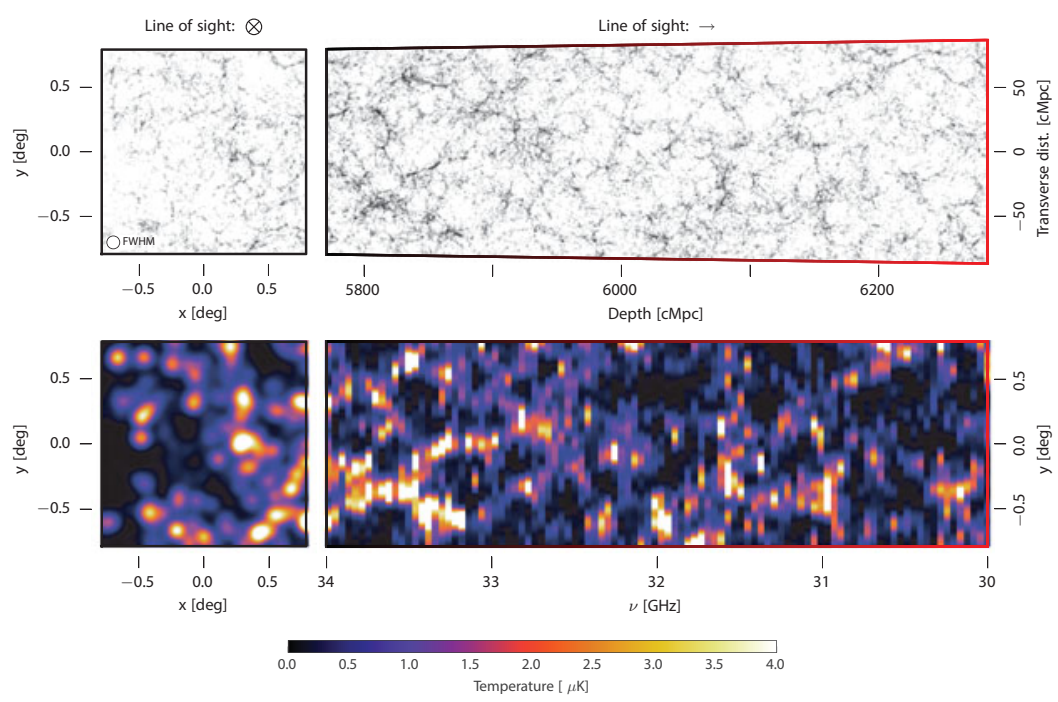

Figure 3. Simulated 3D volume as observed by the COMAP CO intensity mapping experiment; figure and caption are reproduced from Li et al. (2016). (Top:) 2D projections of halo positions. The left image shows the "front" view of halos that would fall into the highest frequency channel, or lowest redshift slice. The COMAP pathfinder beam size is shown for scale. The right image shows the "side" view of halos to a depth of 6 arcmin, or one beam width. (Bottom:) CO intensity map produced by the fiducial model of Li et al. (2016). The same large-scale structure is readily apparent in both images, even with the lower resolution of the intensity map. Note the colour scale ranging between 0 and $4 \mu \mathrm{K}$.

differences between bolometers into the noise component, which then subsequently were spread around the sky by the noise filter, resulting in what were internally in Planck referred to as "dolphins". This effect could then be improved by introducing various bandpass corrections. Similarly, the effects marked by (3) and (4) could be associated with sidelobe and transfer function non-idealities, respectively.

We believe this simple example provides the single most clear demonstration of the strength of the Bayesian approach to component separation available today: By forcing oneself to work within a physical and rigid model at the level of the raw data, in which no arbitrary parameters or "fudge factors" are allowed, even subtle non-idealities are revealed through statistically rigorous goodness-of-fit tests and residual maps. Furthermore, these tests provide far more intuitive information than methods that, say, live purely in power spectrum domain, as it is much easier to visually identify a given systematic effect in terms of residual map than a power spectrum. As is often stated, "seeing is believing", and that holds very true for high signal-to-noise data analysis.

\section{Prospects for Bayesian analysis of intensity mapping experiments}

As the cosmology community is gearing up towards mapping the full $3 \mathrm{D}$ distribution of matter in the universe, the intensity mapping approach has appeared as a particularly promising technology for surveying large volumes in short time. Many of the observational techniques required for this are similar to those used for CMB analysis, and it is therefore appropriate to ask whether also the Bayesian approach described above may turn out to be valuable for intensity mappers.

To answer this question, we believe that the Planck experience may provide some useful guidance. And the most important lesson learned from that work is that the importance 
of full joint and physical analysis increases with the signal-to-noise ratio of the data set in question. When the data are largely dominated by "white noise", the substantially higher computational and implementational cost of a Bayesian approach is unlikely to be justified; it does take longer to write a full-blown Gibbs sampler than an internal linear combination method, and the results are very comparable for noise-dominated data. However, when the data eventually mature, and the signal-to-noise ratio increases, more and more systematic effects become visible above the noise floor, both in the form of astrophysical and instrumental effects - and in particular in terms of interplay between the latter two. And it is in this regime that the Bayesian approach is uniquely powerful.

We are personally involved in the CO intensity mapping experiment called COMAP (Li et al. 2016), which will start observations in early 2018. It will field 19 coherent detectors on a 10-meter telescope, each one coupled to a ROACH that provides a spectral resolution of up to 4096 frequencies between 26 and $34 \mathrm{GHz}$. In total, this setup will cover redshifts between $z=2.4$ and 3.4. The first observations are, however, designed as a pathfinder, and the expected random noise after 2 years of integration is $\sim 10 \mu \mathrm{K}$ per resolution element. For comparison, the bottom panel of Figure 3 shows the predicted CO signal for a slice through the 3D volume, as simulated by Li et al. (2016); note that the colour range spans from 0 to $4 \mu \mathrm{K}$. Thus, the signal-to-noise ratio will be no larger than 0.4 per resolution element. In this situation, a global Bayesian approach is unlikely to yield any significant advantages over far simpler and computationally cheap approaches.

However, in the second phase of the COMAP experiment, the plan is to expand the array by a factor of six in the number of detectors, and increase the observation time to at least three years, leading to an increase in sensitivity per resolution element of at least $\sqrt{1.5 \cdot 6}=3$. The signal-to-noise ratio will then approach unity, and at that stage it becomes important to properly account for subtle instrumental effects.

Based on our experience with Planck, we are in COMAP currently working toward a simple and computationally fast analysis framework for the first phase of the experiment, but at the same time we are planning for a 3D version of Commander that will be suitable for the second phase of COMAP. While the intensity mapping field clearly is in its infancy today, we do believe that it is only a matter of time before we will see high signal-to-noise data coming out of these experiments as well, and then the methods described will be essential. Furthermore, from the Planck experience, we know that it takes about a decade to make such an approach work properly, and so it is probably wise to get started.

\section{References}

Eriksen, H. K., O'Dwyer, I. J., Jewell, J. B., et al. 2004 ApJS, 155, 227

Eriksen, H. K., Jewell, J. B., Dickinson, C., et al. 2008, ApJ, 676, 10-32

Jewell, J. B., Levin, S., \& Anderson, C. H. 2004, ApJ, 609, 1

Leach, S. et al. 2008, A\&\&A, 491, 597

Li, T. Y., Wechsler, R., Devaraj, K., \& Church, S. E. 2016, ApJ, 817, 2

Planck Collaboration 2016, $A \& \mathcal{E} A, 594$, A1

Planck Collaboration 2016, $A \& A$ A, 594, A8

Planck Collaboration 2016, $A \& A$, 594, A10

Planck Collaboration 2016, A\& $A, 594$, A13

Planck Collaboration 2016, A\&A, 594, A15

Planck Collaboration 2016, $A \& A$, 594, A17

Wandelt, B. D., Larson, D. L., \& Lakshminarayanan, A. 2004, PhysRev D, 70, 083511 